\title{
A Rare Case of Native Mitral Valve Bacillus Cereus Endocarditis Culminating Into a Cerebrovascular Infarction
}

\author{
Bibai Ren ${ }^{\mathrm{a}, \mathrm{c}}$, Glenmore Lasam ${ }^{\mathrm{b}}$
}

\begin{abstract}
We report a case of a 56-year-old man who presented initially with a sudden onset of right-sided facial droop and weakness, aphasia, and confusion with no associated fever, chills, syncope, fatigue, weight loss, night sweats, nausea, vomiting, diarrhea, odontalgia, palpitations, cough, or dyspnea. Code stroke was called and the patient received tissue plasminogen activator (tPA) with subsequent resolution of his symptoms. Cranial magnetic resonance imaging showed left frontal punctate cortical restricted diffusion consistent with subacute to acute infarction. Transesophageal echocardiogram showed a severely thickened anterior mitral valve leaflet with a shaggy echodensity consistent with a vegetation. Blood cultures grew Bacillus cereus sensitive to clindamycin, trimethoprim sulfamethoxazole, and vancomycin. He was initially treated with ampicillin, clindamycin, and vancomycin and was eventually maintained solely on vancomycin. $\mathrm{He}$ had complete return of his neurological function and was discharged on intravenous antibiotic to complete a 6-week course.
\end{abstract}

Keywords: Bacillus cereus endocarditis; Cerebrovascular infarction; Native mitral valve vegetation; Prolonged antibiotic therapy; Valve replacement

\section{Introduction}

Bacillus cereus endocarditis in a native mitral valve is a rare occurrence, and is an especially uncommon etiology for a cerebrovascular infarction. An immediate intervention is essential to abolish the evolution of symptoms and to avoid detrimental complications linked with this risk factor for a stroke.

\section{Case Report}

A 56-year-old man with no known comorbidities nor previous

Manuscript submitted January 1, 2018, accepted February 8, 2018

aDepartment of Medicine, Overlook Medical Center, Summit, NJ 07901, USA ${ }^{b}$ Department of Cardiology, Morristown Medical Center, Morristown, NJ 07960, USA

${ }^{\mathrm{c} C}$ Corresponding Author: Bibai Ren, Department of Medicine, Overlook Medical Center, Summit, NJ 07901, USA. Email: bibairen@gmail.com

doi: https://doi.org/10.14740/cr672w surgical operations, noncontributory family profile, and nonsignificant social history presented to the hospital because of a new onset of right-sided facial droop and weakness, aphasia, and confusion. He denied any fevers or chills, syncope, fatigue, weight loss, night sweats, nausea, vomiting, diarrhea, odontalgia, palpitations, cough, or dyspnea. He has no history of intravenous drug use. Of note, he had a tick bite about 2 weeks prior to admission.

A code stroke was called on presentation to the emergency room and the patient received tissue plasminogen activator (tPA) and subsequently regained his lost functions thereafter. He was hemodynamically stable with no note of distress. Physical examination showed no obvious poor dentition, skin findings consistent with intravenous drug use, or signs of endocarditis such as petechiae, splinter hemorrhages, or lesions on the palms or soles. There was no carotid bruit, irregular heart rhythm, or cardiac murmurs appreciated. Neurologic examination post tPA administration showed intact mentation and orientation, functional cranial nerves, preserved motor strength, and normal cerebellar status. Comprehensive metabolic panel, hemogram, lipid panel, and glycosylated hemoglobin were within normal. Hypercoagulability workup was negative. Ancillary tests, which include antineutrophilic antibodies, antinuclear antibody, anticardiolipin antibody, syphilis antibody immunoglobulin $\mathrm{IgG}, \mathrm{SSA}$ and SSB autoantibodies, and Lyme disease antibodies were all negative as well. Twelve-lead electrocardiogram including telemetry demonstrated sinus rhythm with no ectopy. Chest radiograph revealed no acute pulmonary findings. Brain magnetic resonance imaging revealed a left frontal punctate cortical restricted diffusion with probable subtle associated T2 hyperintensity consistent with subacute to acute infarction (Fig. 1a) Transesophageal echocardiography showed mild to moderate mitral regurgitation and severely thickened anterior mitral valve leaflet with a shaggy echodensity (about $6 \mathrm{~mm}$ ) with very small mobile components consistent with a vegetation (Fig. 1b).

Blood cultures were obtained daily until no growth was shown, and for the first 3 days, consistently showed growth of Bacillus cereus sensitive to clindamycin, trimethoprim sulfamethoxazole, and vancomycin. He never developed a fever, leukocytosis, or had an elevated acute phase reactants during his hospital stay. He was initially treated with ampicillin, clindamycin, and vancomycin and was eventually maintained solely on vancomycin based on sensitivities. He had complete resolution of his neurological function and was discharged home with intravenous vancomycin to complete 6-week course. 


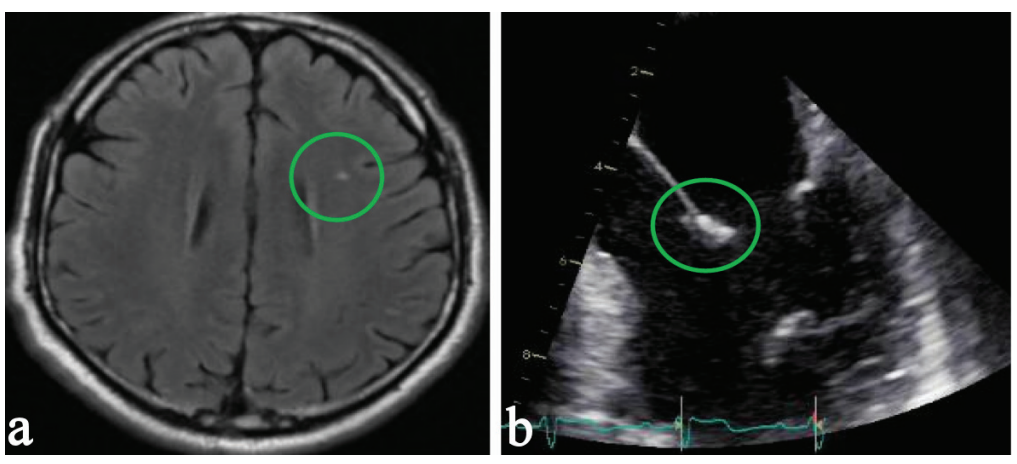

Figure 1. (a) Brain magnetic resonance imaging revealing left frontal punctate cortical restricted diffusion with probable subtle associated T2 hyperintensity consistent with subacute to acute infarction. (b) Transesophageal echocardiography showing a severely thickened anterior leaflet of the mitral valve with a shaggy echodensity consistent with vegetation.

\section{Discussion}

Bacillus cereus is an aerobic-to-facultative, Gram-positive spore forming rod that is widely found in the environment. The organism can cause food-poisoning-related illness or nongastrointestinal disease. Gastrointestinal illnesses has been attributed to the heat-stable preformed toxins that has been implicated as the cause of nausea and emesis particularly after consuming rice or other starchy food [1]. Nongastrointestinal Bacillus cereus infections can present as pneumonia, meningitis, encephalitis, or brain abscess [2].

Endocarditis due to Bacillus cereus is very rare. It is especially observed in intravenous drug users, patients with an underlying valvular pathology, or intravascular appliance such as pacemakers and intracardiac prosthetic valves [3]. Right-sided vegetations have been attributed to intravenous drug use while left-sided vegetations were mainly described from prosthetic valves or implanted devices [4]. Apparently, heroin intravenous drug users have predilection to tricuspid valve endocarditis compared to non-heroin intravenous drug users [5].

There has been no previously reported clinical vignette of Bacillus cereus endocarditis of the native mitral valve in the absence of predisposing factors. However, few cases had been reported in literature of patients with mechanical heart valve with documented Bacillus cereus endocarditis [1]. Much more, there have been no precedent reports of cerebrovascular accident in patients with native mitral valve Bacillus cereus endocarditis without any symptoms such as fevers, or laboratory findings such as leukocytosis or elevated acute phase reactants. Nevertheless, Bacillus cereus aortic native valve endocarditis with multiple brain infarctions had been documented [6].

The portal of entry of the bacteria in our vignette was likely the skin because of the patient's reported tick bite. An isolated case of an acute Bacillus cereus mitral valve endocarditis of an immunocompromised young man with an acute lymphoblastic leukemia that has an ulcerating cutaneous lesion has been reported [7]. The native mitral valve involvement in our case points against intravenous drug use because the majority of native valve Bacillus cereus endocarditis in intravenous drug users was linked with right-sided vegetations, which could be the consequence of the bacterial access site from contaminat- ed drug injection paraphernalia. However, venipuncture site trauma with subsequent development of thrombophlebitis had been proposed as the venue for Bacillus cereus entry to the bloodstream in one occasion [8]. Also, colonization of a susceptible cardiac valve by Bacillus cereus can be due to hematological spread of the bacteria that originated from invasion of the gastrointestinal tract under suitable state [9].

The foremost purpose of antibiotic therapy in infective endocarditis is vegetation sterilization to eventually eradicate the infection, thus, a prolonged, parenteral, and bactericidal therapy is necessary for contemplated cure [10]. Bacillus cereus endocarditis is treated for at least 6 weeks with vancomycin or alternative agent to which the isolate is known to be susceptible [11]. Valve replacement in addition to antimicrobial therapy is required for Bacillus cereus prosthetic valve endocarditis [12] while pacemaker-related Bacillus cereus endocarditis requires removal of the pacemaker besides antibiotics [13].

\section{Conclusions}

Meticulous investigation of the etiology of cerebrovascular accident would recognize the culprit for the event, thus, would commence a prompt and proper intervention to deter the progression of an imminent stroke and eventual avoidance of harmful complications. Endocarditis should be considered in patients with positive blood cultures even for rare organisms infecting native cardiac valves.

\section{Conflict of Interest}

The authors declare that they have no conflict of interest.

\section{References}

1. Thomas BS, Bankowski MJ, Lau WK. Native valve Bacillus cereus endocarditis in a non-intravenous-drugabusing patient. J Clin Microbiol. 2012;50(2):519-521.

2. Drobniewski FA. Bacillus cereus and related species. Clin Microbiol Rev. 1993;6(4):324-338. 
3. Logan NA, Popovic T, Hoffmaster A. Bacillus and other aerobic endospore forming bacteria. In: Manual of Clinical Microbiology, Murray PR, Baron EJ, Jorgensen JH, et al (Eds), American Society for Microbiology Press, Washington, DC 2007. 2000. p.455.

4. Ngow HA, Wan Khairina WM. Bacillus cereus endocarditis in native aortic valve. J Infect Chemother. 2013;19(1):154-157.

5. Jain V, Yang MH, Kovacicova-Lezcano G, Juhle LS, Bolger AF, Winston LG. Infective endocarditis in an urban medical center: association of individual drugs with valvular involvement. J Infect. 2008;57(2):132-138.

6. Kitazawa T, Totsuka M, Yoshino Y, Koga I, Ota Y. Bacillus cereus native valve endocarditis with multiple brain infarctions: a case report and a review of the literature. Clin Res Infect Dis. 2015;2(2):1019.

7. Cone LA, Dreisbach L, Potts BE, Comess BE, Burleigh WA. Fatal Bacillus cereus endocarditis masquerading as an anthrax-like infection in a patient with acute lymphoblastic leukemia: case report. J Heart Valve Dis. 2005;14(1):37-39.

8. Castedo E, Castro A, Martin P, Roda J, Montero CG. Bacillus cereus prosthetic valve endocarditis. Ann Thorac
Surg. 1999;68(6):2351-2352.

9. Bottone EJ. Bacillus cereus, a volatile human pathogen. Clin Microbiol Rev. 2010;23(2):382-398.

10. Baddour LM, Wilson WR, Bayer AS, Fowler VG, Jr., Bolger AF, Levison ME, Ferrieri P, et al. Infective endocarditis: diagnosis, antimicrobial therapy, and management of complications: a statement for healthcare professionals from the Committee on Rheumatic Fever, Endocarditis, and Kawasaki Disease, Council on Cardiovascular Disease in the Young, and the Councils on Clinical Cardiology, Stroke, and Cardiovascular Surgery and Anesthesia, American Heart Association: endorsed by the Infectious Diseases Society of America. Circulation. 2005;111(23):e394-434.

11. Noonan L, Freeman J. Bacillus cereus and other non-anthracis Bacillus species. UpToDate, Waltham, MA. (Accessed on November 19, 2017).

12. Steen MK, Bruno-Murtha LA, Chaux G, Lazar H, Bernard S, Sulis C. Bacillus cereus endocarditis: report of a case and review. Clin Infect Dis. 1992;14(4):945-946.

13. Abusin S, Bhimaraj A, Khadra S. Bacillus Cereus Endocarditis in a permanent pacemaker: a case report. Cases J. 2008;1(1):95. 